\title{
EFFORT-D study process evaluation: challenges in conducting a trial into the effects of running therapy in patients with major depressive disorder
}

\author{
Frank Kruisdijk ${ }^{1,2^{*}}$ (D) Ingrid Hendriksen ${ }^{2,3}$, Erwin Tak ${ }^{2,3}$, Aart-Jan Beekman ${ }^{4}$ and Marijke Hopman-Rock ${ }^{2,3,5}$
}

\begin{abstract}
Background: Exercise is currently seen as an effective treatment for major depressive disorder (MDD). However, existing studies have focused mainly on mild-to-moderate depression. The moderate positive effect of exercise found in meta-analyses concerning these studies differs, however, from the harsh daily clinical practice, when trying to implement exercise as an adjunctive treatment. We aimed to evaluate the feasibility of aerobic exercise in MDD and identify future problems for implementation.
\end{abstract}

Methods: The EFFect Of Running Therapy on Depression (EFFORT-D) study was a randomized clinical trial examining the effectiveness of running therapy or Nordic walking in inpatients and outpatients with MDD. We conducted a process evaluation based on the method of Linnan and Steckler. Participant inclusion, dropout and no show were registered qualitatively and quantitatively.

Results: The inclusion and delivered dose of the exercise interventions were limited ( 60 and $75 \%$, respectively), leading to $80 \%$ less inclusion than foreseen. Motivational doubts were the main reason not to participate in the study. The unexpected high dropout rates (40\% after 3 months and $80 \%$ after 12 months) were frequently related to lack of motivation due to disease characteristics and severity. The duration of the intervention, longer than 3 months was another underlying factor for poor adherence.

Conclusions: Depression severity appeared to be the key factor determining dropout, followed by the duration of exercise intervention, expressed by a pre- and post-inclusion lack of motivation. Both running therapy and Nordic walking were apparently unsuitable for most patients with MDD in the current format. Emphasis on motivational issues is necessary from the early start of the intervention in these patients with MDD. Also a tailored and steppedcare approach is advised for future implementation.

Trial registration The randomized controlled trial protocol of EFFORT-D was approved by the Medical Ethical Committee for Mental Health (Metigg Kamer Noord), CCMO (Central Committee on Research Involving Human Subjects) Protocol Number: NL.26169.097.08. Registration in the Netherlands Trial Register (NTR): NTR1894 on July 2, 2009

\section{Background}

Major depressive disorder (MDD) has huge effects on wellbeing and daily personal and professional functioning. It is the second leading cause of disease burden based

\footnotetext{
*Correspondence: f.kruisdijk@planet.nl

1 Department Innova, GGz Centraal Innova, Amersfoort, The Netherlands

Full list of author information is available at the end of the article
}

on years lived with disability [1], and the loss of dailyadjusted life years is considerable [2]. Depressive symptoms are common in various populations [3]. Recurrence of symptoms of MDD occurs in an unfavourable but fluctuating course in $44 \%$ of the patients and a severe chronic course in $12-32 \%$ of the patients $[4,5]$. Pharmacotherapy and psychotherapy, i.e. cognitive behaviour therapy and interpersonal therapy, are the mainstays of treatment $[6$, 
7]. Effective use of antidepressant medication is often compromised by side effects, while both the availability of trained therapists and lack of motivation on the part of patients may limit the utility of antidepressant psychotherapy [8]. Although for instance cognitive therapy treatment has a positive effect, about $50 \%$ of the patients relapse after 2 years with an estimated $80 \%$ relapse after 5 years [9]. Meta-analysis of standard MDD treatment showed a clinically and statistically heterogeneous outcome of treatment as usual: about $30 \%$ of the patients remitted from depression, but depressive symptoms also worsened in $12 \%$ [10].

Exercise is currently seen as a promising alternative to pharmacotherapy and psychotherapy, because it has low costs and few side effects, and is also considered safe for most patients. Previous research showed that a low level of physical activity is highly prevalent in individuals with depression [11] and that exercise has a positive effect on physical health in such a population [12-14]. Physical activity also has additional advantages in the large group of patients that suffer from a combination of mental and physical problems, such as depression and diabetes, in which prevention of metabolic syndrome by enhancing physical activity is vital $[15,16]$.

Using exercise therapy as (part of the) regular treatment for MDD seems, therefore, a logical consequence. However, studies focussing on exercise therapy as (part of the) treatment for depression usually involved patients with mild to moderate depression. These patients were mostly recruited through public announcements, and attracted participants who are different from the population with MDD that is hospitalised in recognised mental health care institutions. Of the 28 studies selected by Mead et al. [17] in their Cochrane review on exercise and depression, only two were randomized controlled trials (RCTs) that included adult inpatients $[18,19]$. In a follow-up Cochrane review of Cooney et al. [20], only seven of 39 studies recruited participants from clinical populations, i.e. hospital inpatients or outpatients referred by a general practitioner (GP). The studies included in the latter review, that reported positive effects on quality of life and symptom severity in severely depressed inpatients, were all short-term studies.

One of the few studies into severe MDD inpatients, which was not included in the aforementioned Cochrane reviews, had an add-on design with, besides exercise, treatment as usual consisting of pharmacotherapy and/ or electroconvulsive therapy [20]. Although this study lasted only 2 weeks, it showed a significant effect on depressive symptoms as well as physical and psychological domains of quality of life; however, $50 \%$ of the eligible patients were not motivated to participate. The eight trials with a longer follow-up in the Cochrane review of
Cooney et al. [21] using an intervention time between 10 and 16 weeks and follow-up measurements with a maximum of 10 months, showed only a moderate effect on depression with a pooled standardised mean difference (SMD) of -0.33 . This is in line with the review of Krogh et al. [22] studying RCTs in adults with a clinical depression. This review showed a significant SMD of -0.40 in 13 trials, with an inverse relationship between duration of the intervention and the magnitude of the association between exercise and depression. Also, a pooled analysis of five trials with a long-term follow-up (9-26 months), suggested no long-term benefit (SMD - 0,01). Consequently, when implementing exercise in for instance a guideline for treatment of MDD, a diminishing effect of exercise in the long run should be considered.

Limited evidence is found that short-term interventions are as effective as long-term interventions in MDD [23]. The latest review in this field advised moderate exercise to be suitable as part of a multidimensional approach [24]. A limitation of existing studies is that bias must be considered regarding inadequate blinding of participants and observers. When studies with adequate allocation concealment, intention-to-treat-analysis and blinded outcome assessment were analysed, the pooled SMD $(-0.18)$ for the primary outcome of depression scores was small and no longer significant $[17,21,25]$.

An important aspect of MDD is the unpredictable duration of a depressive period. In an epidemiological cohort study, half of those with MDD and a minor depressive episode recovered within 6 months with standard treatment, wherein only a short period without treatment seemed, however, defensible. Treatment was urgently advised for those with greatest need, a lower functioning at onset and experiencing comorbidity [5]. However, it is unknown how long an intervention should take. The abovementioned controversy between moderate positive results from meta-analyses and treatment of severe (inpatient) MDD in the harsh clinical daily practice still exists, leaving clinicians and patients uncertain about the optimal use of exercise as a part of their treatment. As a result, more high-quality research into the relationship between MDD and exercise as a (add-on) treatment was needed.

The EFFect Of Running Therapy on Depression (EFFORT-D) study, which has been described in a previous publication, was both aimed at severe (inpatient) MDD and a long-term exercise intervention in an addon RCT design [26]. During the EFFORT-D study, which was carried out between 2012 and 2015, both an effect evaluation (results to be published) and a process evaluation were conducted. The process evaluation enabled us to determine influencing factors to the outcome results in different phases and identify topics for future research, 
such as implementation strategies in clinical practice and advices for depressive patients. To our knowledge, only few process evaluation studies in this field have been published [27-29]. The importance of a process evaluation has been demonstrated by Linnan and Steckler [30]. Although their examples focused on preventive population interventions, their framework can also be applied to our study focusing on curative interventions for patients. Therefore, the aim of this process evaluation was to gain insight into the context, reach and dose of the exercise intervention of the EFFORT-D study.

\section{Methods EFFORT-D study \\ Aim}

The main objective of the EFFORT-D study was to assess the effectiveness of exercise therapy [running therapy (RT) or Nordic walking (NW)], in addition to usual care, on MDD in adult (in) patients. We hypothesised that adding exercise therapy to usual care would result in a larger reduction in depressive symptoms [as measured with the Hamilton Rating Scale of Depression (HAM$\left.\mathrm{D}_{17}\right)$ ], during a 6-month treatment programme as well as at 6 months follow-up, compared to usual care without exercise therapy.

\section{Design}

EFFect Of Running on Depression was an observerblinded randomised controlled parallel trial conducted in three Dutch mental health care hospitals between December 2012 and January 2015. Participants were randomly assigned to 6 months of RT or NW twice a week in addition to their treatment as usual. Both groups were followed during 1 year with measurements at inclusion (T0), 3 months (T3), 6 months (T6) and a final after 12 months (T12).

It was expected that patients in the usual care group (control group) would respond with a mean reduction in HAM- $\mathrm{D}_{17}$ of six points, because they were all following the standard MDD treatment protocol. Adding exercise to usual care (intervention group) was expected to result in a decline of at least eight points on the HAM-D ${ }_{17}$ score. To detect this difference, with an $\alpha$ (two-tailed) of $5 \%$ and a power $(1-\beta)$ of $80 \%$, using two equal groups and a standard deviation of 5 points, 100 patients were needed in each group. Taking 30\% dropout into account, 140 patients had to be included in each group.

Because EFFORT-D focussed on patients with MDD, an add-on design with exercise was indicated due to ethical considerations: no active treatment at all versus exercise was no option due to the nature, type and severity of the participant's MDD.

\section{Participants}

The study was performed in three regions of a large specialised mental health care institution. Included patients were either inpatients, day-hospital patients or outpatients referred by specialised mental health care such as emergency services (in- and day-hospital patients) and Dutch general practitioners (GPs) for outpatients.

The study population of EFFORT-D consisted of patients with MDD with a Hamilton depression scale (HAM- $\mathrm{D}_{17}$ ) score $\geq 14$, indicating at least a moderate depression at the time of inclusion. Because the GPs already had started with the depression protocol several months before the start of this study, the depressive symptoms of these outpatients were usually in partial remission.

\section{Measurements and procedure}

The control group received treatment as usual, which consisted of pharmacotherapy, supportive psychotherapy and daily activation for the hospitalized participants or those in-day treatment, and pharmacotherapy and cognitive therapy for the outpatients. The experimental group received an exercise intervention in addition to treatment as usual. This consisted of a group-based Running Therapy (RT) or Nordic Walking (NW) session of $1 \mathrm{~h}$, twice a week for the duration of 6 months, with increasing intensity following a training protocol with the possibility for individual adaptations. The control group was only allowed to practice low-intensity, non-aerobic exercises. Participants were monitored closely: no shows and dropouts were registered including the arguments of the participant. Participants were followed up using the Hamilton Depression Scale (HAM-D ${ }_{17}$ ) scores, the Astrand submaximal cycling test and questionnaires at 3, 6 and 12 months (T3, T6 and T12, respectively). All participants gave their informed consent for participation in the study.

\section{Methodological framework of this process evaluation}

In their model, Linnan and Steckler mentioned several factors that are important in a process evaluation [30]. In the current process evaluation, we used the factors context, reach and dose from their model, because these were assumed to be the most suitable to evaluate our intervention study:

- Context refers to aspects of the larger social, political and economic environment that may influence the intervention.

- Reach describes the proportion of the intended population that participates in the intervention or study. 
- Dose, which consists of dose delivered and dose received, measures the intended intervention and the extent to which participants adhere to it.

Furthermore, the exercise trainers and the research assistant were asked by a questionnaire to answer nine open questions for evaluation purposes. These questions were based on the context, reach and dose factors as defined by Linnan and Steckler, along with two general evaluative questions. Answers were received by email and summarised by the first author.

\section{Results}

\section{Context}

Much less than the required 200 subjects (100 in both the intervention and control group) was included. The financial crisis and the associated changes in the Dutch mental healthcare system had a major-and unexpected-impact on the social, political and economic environment in which this study took place. As a result, the recruitment of patients was restrained. Regarding the inclusion sites, $17 \%$ of the participants were initially assigned to a locked emergency ward. After stabilization and reduction of suicide risk, they continued their stay voluntarily in an open crisis ward. They were invited to participate in the study after a clinician indicated that they could give informed consent; they often continued their treatment in a day hospital followed by an outpatient programme. The other inclusion sites were day hospitals as a start of the treatment and outpatient clinics. Finally, $45 \%$ of the included participants were treated MDD inpatients or were day-hospitalized.

\section{Reach}

Due to the abovementioned developments at the start of the study, the reach was severely reduced. An important contextual reason for reduced inclusion was the exclusion of three of the original five study locations. Budgetary cuts and strategic decisions about the affective disorders programme were underling arguments. The previously estimated reach in the population was approximately 3500 patients in a programme for depressive disorders and about 500 patients from emergency wards. With the loss of three locations, the reach was reduced to approximately 1200 patients in a programme and about 200 patients from emergency wards, which caused a decrease of approximately $60 \%$ potentially to reach. Table 1 shows the demographic characteristics of the included participants.

\section{Dose-related findings}

In total, 183 patients were screened for eligibility to participate within the 2-year time-frame of the EFFORT-D

\begin{tabular}{ll}
$\begin{array}{l}\text { Table } 1 \text { Baseline } \\
\text { of the participants }\end{array}$ & demographic \\
\hline & $\boldsymbol{N}=\mathbf{4 8}$ \\
\hline Age, years (SD) & $41.6(9.1)$ \\
Female, \% & 62.5 \\
Education, \% & PS: 5; SecS: 30; LVE/MVE: 28; HS/HVE/acad: 37 \\
Country of origin, \% & Netherlands: 79 \\
& Turkey/Morocco: 8 \\
Civil status, \% & M: 13 \\
\hline
\end{tabular}

PS primary school, SecS secondary school, LVE lower vocational, MVE medium vocational, HS/HVE/acad high school/higher vocational/academic, $M$ married, $S$ single, $D$ divorced

a Include Surinam, Aruba, Curacao and Iraq

study. Ultimately only 48 participants could be included in the study, with 25 participants in the intervention arm, instead of the required 100 participants in each group. The relatively high number of eligible participants that were excluded $(n=135)$ was due to several causes. First, $20 \%$ of the patients referred by a GP were admitted to the affective disorder programmes after completing the intake procedure, but by the time they were rated on the HAM-D ${ }_{17}$, their cut-off score was below 14 , which made them ineligible to participate. Their HAM$D_{17}$ score was probably higher before referral to specialist care, but standard treatment was already initiated before the research assistant could make a screening appointment to measure the HAM- $\mathrm{D}_{17}$. Other causes for exclusion were other health problems during screening (14\%), already exercising twice a week for at least $30 \mathrm{~min}(14 \%)$ and a contraindication for the intervention given by the primary treating psychiatrist (12\%). The largest barrier to inclusion, however, resulted from the large proportion of patients $(40 \%)$ who expected that they were not able to organise their daily life in such a way that they could participate in the exercise intervention twice a week. The programme was too time consuming, they were too tired and, in their own opinion, were unable to invest in exercise and commitment to the study. These reasons were assumed to be associated with MDD symptoms as fatigue, loss of energy and initiative. The planned dose of exercise was finally delivered to 25 participants in the intervention group, of which $45 \%$ were inpatients of an emergency ward or were being treated in a day hospital. As expected, due to the severity of their depression, the average HAM- $D_{17}$ score at inclusion for inpatient or day hospital participants (25.7) was higher than for outpatients (20.5). The patient flow during all measurements (T0, T3, T6 and T12) is presented in Fig. 1.

Between allocation to the intervention group (T0) and the first follow-up measurement (T3), already 10 


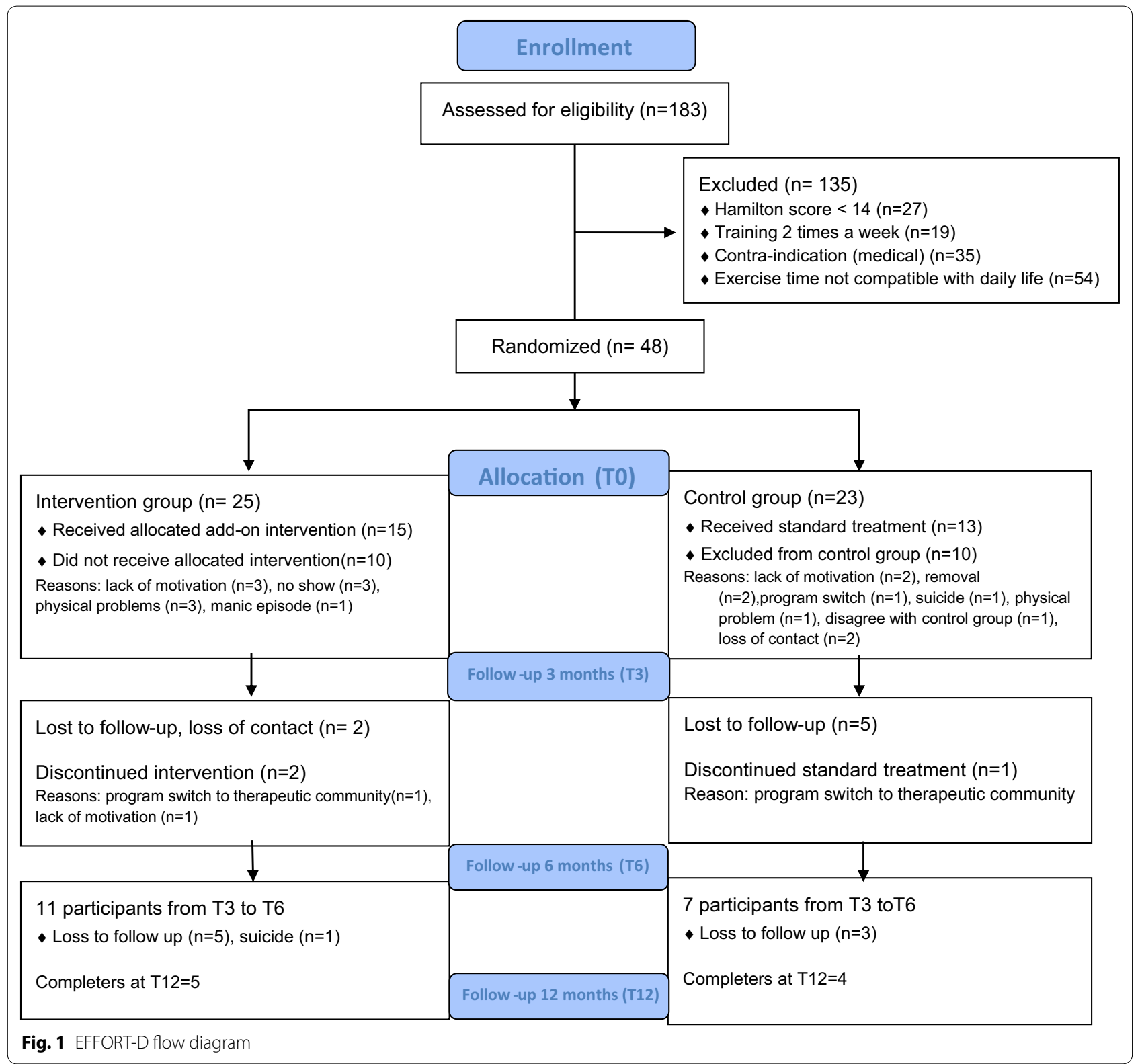

of the 25 participants (40\%) dropped out. This dropout increased to $56 \%$ at $\mathrm{T} 6$ and $80 \%$ at T12. In the non-intervention arm the same pattern was seen. For the drop-out reasons see Fig. 1.

\section{Evaluation of the nine-item questionnaire}

We decided not to contact the participants at the end of the study for the process evaluation. Participants who dropped out could not easily be contacted by the research assistant, and the small proportion of participants who reached the T12 evaluation (20\%) in the intervention arm could cause bias in favour of the RT and NW activities. The answers to nine open questions showed that the trainers $(n=2)$ and the research assistant had positive attitudes about their role in the study.

However, they all reported that it was unexpectedly difficult to motivate the participants. The expectation of a positive effect of the RT and NW was still present, but trainers and research assistant developed a more realistic view on the feasibility of implementing RT and NW in these depressive patients. The many dropouts at every stage of the study compelled them to be cautious for any positive expectations. According to the exercise trainers (see questions described in Table 2), the 'ideal patient' who can benefit from RT or NW is a female patient 
Table 2 Evaluation questionnaire for exercise trainers and the research assistant

\begin{tabular}{ll}
\hline Context-related questions & What aspects went well during the study? \\
Reach-related questions & What would have been the ideal environment for the study? \\
& Did the participation in the study meet your expectations? \\
Dose-related questions & Describe the profile of a patient suffering from depression who improves with exercise \\
& Describe the profile of a patient for whom exercise will have little effect \\
General questions about context, reach and dose & Estimate the percentage of participants in the intervention arm that completed the intervention \\
& Did the participation in the study meet your expectations? \\
& What needs improvement? What advice can you give to other researchers studying exercise in \\
& depressive patients in the future?
\end{tabular}

between 30 and 50 years old, who is native Dutch and has previous positive exercise experiences with running.

This positive exercise experience could have been a long time ago, but could be reactivated during the RT and/or NW intervention. For depressed inpatients who are not native Dutch, middle aged, male and with no exercise experience, a very modest form of walking without training goals was reported to be most feasible. According to the exercise trainers, the inpatients were serious about participating, but training two times a week according to the protocol was ultimately too difficult for these patients to maintain. Even when the programme was adapted, without violating the protocol, whereby patients were coached individually and started with gradual walking exercises, the participants could not keep up participation. For outpatients, who had to travel to the intervention site, the intervention often was too time consuming. At the time the patients started the first series of training, their motivation to complete the intervention was considered high by the trainers and the research assistant, so during the intervention it appeared difficult to maintain their motivation. When asked to suggest improvements for the study, the trainers and research assistant referred to a shorter intervention period, with a suggested maximum of 3 months. The trainers indicated that this could have a positive effect on the inclusion because it will be easier to motivate the patients with a time horizon of 3 months, and at the same time the costs for trainers could be reduced. In addition, they suggested that a shorter travel distance to the intervention site could have a positive effect.

Another constraint of this research, according to the trainers, was the winter weather in the Netherlands. To complete the exercise intervention, dry weather and moderate temperatures were seen as ideal. Indoor training was not preferred, because the ideal training environment for RT or NW is in the woods or at the countryside, but indoor facilities are necessary for the continuity of the activities. In this study enduring wintry conditions caused too much discontinuity in the trainings scheme.
When asked to estimate the percentage of participants that would complete the intervention, provided that the first sessions were finished, the trainers indicated $70 \%$, while the research assistant indicated only $30 \%$. To prevent potential demotivation of the research assistant, he was intensively coached by the project leader during the rest of the study. Furthermore, the trainers and research assistant both indicated that communication between them was lacking at several times during the intervention period. The fact that one exercise site was in another region as the research centre was an important causal factor.

\section{Discussion}

This process evaluation showed that economic conditions had an important negative influence on the implementation of the EFFORT-D study. A severely reduced reach, combined with a large loss of dose delivered was shown. Once included in the intervention group, it turned out to be very difficult to motivate patients with MDD to participate in the aerobic exercise therapy and the long-term measurements. The duration of the intervention seemed to be too long according to the evaluative questionnaire. During this study, the research team faced substantial challenges in several areas. An unexpected high proportion (almost three quarters) of the eligible participants was excluded before randomization. As a result, only about one-fifth of the intended number of patients could be included in this study. Also, contextual difficulties, due to an organisational merger and an economic-driven reorganisation, reduced the reach and the number of available patients in the research population. Forty percent of the eligible participants reported that they were unable to incorporate the exercise programme in their daily lives. They argued that they were too tired and the required investment in time was too high. This can be interpreted as a lack of motivation and it seems to form a structural problem for implementation of exercise in MDD. This motivational issue was also found to be a perceived barrier to exercise in another qualitative study 
[27]. Patients in that study were screened for preferences of exercise as a treatment for depression. They reported mood problems, tiredness and lack of motivation as the biggest barriers for exercise in both men and women. In another systematic review on motivating factors and barriers towards exercise, mood problems and stress were also identified as major barriers for engaging in exercise during severe mental illness [31]. Comparable trials in MDD inpatients or severe depressed outpatients who were referred by GPs or psychiatrists also show $25-50 \%$ of the eligible participants that refuse to participate. This may be an indication that severity of depression might be the underlying factor for refusal [20,32]. However, severity of depression seemed not to be an important factor of non-adherence to exercise in a pooled analysis from two clinical trials [33]. This result could be explained by the fact that a population with mild to moderate depression was included in these trials. Finally, in $20 \%$ of the outpatients referred by a GP in our study, depressive symptoms fell below the required threshold for inclusion during the intake procedure. This was possibly due to the mechanism of spontaneous recovery [34] or was a result of treatment that was already started by the GP. The fact that the dropout rate in both arms of our study was comparable could indicate that the intervention itself was not the main cause of the high dropout. It might also mean that strain of the follow-up testing was too high for these severely depressed participants. This was, however, not confirmed by the evaluative questions answered by the exercise leaders and research assistant. The follow-up arguments for quitting the study showed that participating in the early stages of the intervention did not provide enough incentive to remain on board. These kinds of motivational problems are also described in patients with affective disorders such as MDD, bipolar disorder [35] and with schizophrenia [36].

The concept of 'autonomous motivation' to exercise, meaning an intrinsic motivation and an identification with the benefits of a healthy lifestyle, seems to play a vital role in maintaining involvement in the exercise programme. Somewhat hopeful here is the fact that $14 \%$ of our eligible participants were excluded because they already trained twice a week for $30 \mathrm{~min}$. Possibly, the use of a depression guideline of the GPs explains this effect, because it recommends prescribing exercise for mild to moderate depression. It seems that the EFFORT-D intervention and study design in its current form does not seem to be feasible in these MDD patients, certainly not for hospitalised inpatients. So, regarding implementation of exercise programmes in the treatment of MDD, motivational themes need a lot of attention throughout the programme. This is very important, because people with severe psychiatric disorders, such as MDD, are at higher risk for chronic physical conditions [37-39] and tend to be very sedentary [40]. It might seem more adequate to treat MDD patients using the activation principles of patients with other severe mental illnesses such as schizophrenia or bipolar disorder. As described in recent publications, aiming the exercise intervention on improvement of quality of life and cardiorespiratory fitness, besides the improvement of depression, is indicated $[11,38,41]$. A broader multidisciplinary lifestyle intervention, incorporating light-to-moderate physical activity in daily life, in addition to lifestyle interventions aimed at smoking and eating habits, seems effective. A disadvantage of such a multidimensional approach is that it will be difficult to determine the effect of the physical exercise separately, but an advantage is a probably broader health gain. An alternative approach might be indoor training, as used in other studies of depressed patients [32, 42] and in studies of patients with schizophrenia [43]. This could also prevent training absences during wintry conditions with icy running tracks. However, a combination of indoor and outdoor exercise is preferred due to indications that a green environment contributes to wellbeing $[44,45]$. Another option is to launch a pre-study training, in which participants are given the opportunity to personalise their programme with help from a physical therapist with the aim of increasing their fitness level and preventing injuries. This is in line with recommendations based on a qualitative study of patients' viewpoints in general practice involving 33 participants [28]. Once participants have a sufficient fitness level, they can participate in the group intervention. An advantage of this personalised approach is that the form of exercise can be adapted to the patients' preferences and possibilities, for instance cycling in the case of joint problems. In addition, also E-learning environments, supporting technical tools such as electronic devices for self-registration of activity and smartphone applications (including WhatsApp and social media) can be considered. These can potentially encourage participants in daily life and promote more adherence to exercise [46].

Prevention of early dropouts needs more attention in future research. It is a paradox, however, that the estimated length of an optimal exercise study is $3-4$ months, whereas the preferred length of an exercise programme is longer. A single study, with a long follow-up of 10 months, showed that it is worthwhile to invest in long-term motivation to persist in exercising because of maintenance of the therapeutic effect and prevention of a relapse [47]. The latest results of the Dutch NEMISISstudy show that the median time for a depressive episode was 6 months in MDD and 3 months for a minor depressive episode with a full remission of $75 \%$ after 1 year for both [5]. This means that exercise as an adjunctive 
treatment probably can contribute to remission in the first 6 months, after this period it is probably meaningful to prevent a relapse.

This process evaluation had several limitations. First, evaluative questioning of participants was not appropriate, because the expected bias in favour of exercise was estimated to be high: practically only the very small group of completers of the intervention at T12 were able to give feedback. This diminished potential informative and qualitative suggestions for future research from a patient perspective. In particular, evaluation of the larger group of dropouts could have given more detailed information about blockades for exercise or suggestions to solve these, but that was not feasible. Second, the questions directed at the trainers and research assistant for this process evaluation were sent by e-mail. Instead, a (semi)-structured interview would have led to more in-depth information. At last the numbers of included patients were small so the conclusion that this type of exercise in these particular patients does not fit MDD patients cannot be generalised easily. Nevertheless, this process evaluation of an exercise intervention in patients with MDD is one of the few delivered and may be of value for both researchers as well as clinicians in the field. Researchers can profit from the described pitfalls, whereas the clinicians may recognise their own patients and prevent obstacles when planning an (add-on) exercise intervention.

\section{Conclusions}

Our process evaluation showed that both the intervention as well as the study design of EFFORT-D were not feasible for patients with MDD in terms of motivation and compliance. In future interventions, applying electronic self-registration devices is advised, which not only can motivate the participant through direct feedback, but they also enable researchers to objectively measure the intensity and time spent on physical activity. Besides, social media use could enhance more adequate communication (for instance forming an easy accessible WhatsApp or Facebook group). Further research is needed to define the optimal duration of an exercise intervention in MDD. Finally, a tailored and stepped-care intervention method is advised in case of aerobic exercise training such as running therapy or Nordic walking, starting with a pre-study phase in which a physical therapist can prepare the potential participant. Flexibility in aerobic activity fitting the individual participant, and combining in- and outdoor facilities, may also help to retain the participants and enhance motivation for exercise, making it part of their lifestyle.

\section{Abbreviations}

MDD: major depressive disorder; RCT: randomized controlled trial; GP: general practitioner; SMD: standard mean difference; EFFORT-D study: EFFect Of Running on Depression study; RT: running therapy; NW: Nordic walking; HAM-D 17 : 17-item Hamilton Depression Rating Scale; T0, T3, T6, T12: measurements at inclusion, 3, 6 and 12 months.

\section{Authors' contributions}

FK: study design, collection and analysis of data, drafting and revising the manuscript, $\mathrm{H}$ : study design and revising the manuscript, ET: study design and revising the manuscript, $\mathrm{AJB}$ : revising the manuscript, $\mathrm{MH}$ : study design, supporting data analysis and revising the manuscript. All authors contributed to the manuscript. All authors read and approved the final manuscript.

\section{Author details \\ ${ }^{1}$ Department Innova, GGz Centraal Innova, Amersfoort, The Netherlands. 2 Body@Work, TNO-VU University Medical Center, Amsterdam, The Nether- lands. ${ }^{3}$ The Netherlands Organisation for Applied Scientific Research TNO, Leiden, The Netherlands. ${ }^{4}$ Department of Psychiatry, VU University Medical Center, Amsterdam, The Netherlands. ${ }^{5}$ Department of Public and Occupa- tional Health, EMGO Institute for Health and Care Research, VU University Medical Center, Amsterdam, The Netherlands.}

\section{Acknowledgements}

The authors would like to thank all patients and mental healthcare professionals of GGz Centraal who have made efforts to collect all the data, which formed the basis for this publication. Prof. Dr. Erik Knorth for his valuable explanation of the systematic approach of research process evaluations.

\section{Competing interests}

AJB received honorarium as a speaker from Lundbeck (no connection with this study). Other authors: no competing interests.

\section{Availability of data and materials}

The data that support the findings of this study are not publicly available because it is inconsistent with the informed consent. Anonymous data are, however, available from the corresponding author on reasonable request and with permission of GGZ Centraal.

Consent for publication

Not applicable.

\section{Ethics approval and consent to participate}

The EFFORT-D study was designed and implemented in accordance with the principles of the Helsinki Declaration (Edinburgh, Scotland Amendment, October 2000). The study protocol was approved by the Medical Ethical Committee for Mental Health (Metigg Kamer Noord). Centrale Commissie Mensgebonden Onderzoek CCMO (Central Committee on Research Involving Human Subjects) Protocol Number: NL.26169.097.08. All participants gave their informed consent for participation and anonymous publication of the results.

\section{Funding}

Grant support (unrestricted gift) was received from the former "Open Ankh Foundation" (since March 2008 "Zorgcoöperatie Nederland").

\section{Publisher's Note}

Springer Nature remains neutral with regard to jurisdictional claims in published maps and institutional affiliations.

Received: 18 December 2017 Accepted: 19 February 2018 Published online: 24 February 2018

\section{References}

1. Vos T, Flaxman AD, Naghavi M, Lozano R, Michaud C, Ezzati M, Shibuya K, Salomon JA, Abdalla S, Aboyans V, et al. Years lived with disability (YLDs) for 1160 sequelae of 289 diseases and injuries 1990-2010: a systematic analysis for the Global Burden of Disease Study 2010. Lancet. 2012;380:2163-96. 
2. Murray CJL, Lopez AD. Measuring the global burden of disease. N Engl J Med. 2013;369(5):448-57.

3. Salomon JA, Wang H, Freeman MK, Vos T, Flaxman AD, Lopez AD, Murray CJ. Healthy life expectancy for 187 countries, 1990-2010: a systematic analysis for the Global Burden Disease Study 2010. Lancet. 2012;380:2144-62.

4. Beekman AF, Geerlings SW, Deeg DH, et al. The natural history of late-life depression: a 6-year prospective study in the community. Arch Gen Psychiatry. 2002:59:605-11.

5. Ten Have M, Penninx B, Tuithof M, van Dorsselaer S, Kleinjan M, Spijker J, de Graaf R. Duration of major and minor depressive episodes and associated risk indicators in a psychiatric epidemiological cohort study of the general population. Acta Psychiatr Scand. 2017;136:300-12.

6. Cuijpers P, Donker T, Weissman MM, Ravitz P, Cristea IA. Interpersonal psychotherapy for mental health problems: a comprehensive meta-analysis. Am J Psychiatry. 2016;173:680-7.

7. Cuijpers P, Cristea IA, Karyotaki E, Reijnders M, Huibers MJ. How effective are cognitive behavior therapies for major depression and anxiety disorders? A meta-analytic update of the evidence. World Psychiatry. 2016:15:245-58

8. Hollon SD, Munoz RF, Barlow DH, Beardslee WR, Bell CC, Bernal G, Clarke GN, Franciosi LP, Kazdin AE, Kohn L, et al. Psychosocial intervention development for the prevention and treatment of depression: promoting innovation and increasing access. Biol Psychiatry. 2002;52:610-30.

9. Vittengl JR, Clark LA, Dunn TW, Jarrett RB. Reducing relapse and recurrence in unipolar depression: a comparative meta-analysis of cognitivebehavioral therapy's effects. J Consult Clin Psychol. 2007;75:475-88.

10. Kolovos S, van Tulder MW, Cuijpers P, Prigent A, Chevreul K, Riper H, Bosmans JE. The effect of treatment as usual on major depressive disorder: a meta-analysis. J Affect Disord. 2017;210:72-81.

11. Stubbs B, Koyanagi A, Schuch FB, Firth J, Rosenbaum S, Veronese N, Solmi M, Mugisha J, Vancampfort D. Physical activity and depression: a large cross-sectional, population-based study across 36 low- and middleincome countries. Acta Psychiatr Scand. 2016;134:546-56.

12. Blumenthal JA. Effects of exercise and stress management training on markers of cardiovascular risk in patients with ischemic heart disease: a randomized controlled trial. JAMA J Am Med Assoc. 2005;293:1626-34.

13. Bridle C, Spanjers K, Patel S, Atherton NM, Lamb SE. Effect of exercise on depression severity in older people: systematic review and meta-analysis of randomised controlled trials. Br J Psychiatry. 2012;201:180-5.

14. Stubbs B, Rosenbaum S, Vancampfort D, Ward PB, Schuch FB. Exercise improves cardiorespiratory fitness in people with depression: a metaanalysis of randomized control trials. J Affect Disord. 2016;190:249-53.

15. Mezuk B, Eaton WW, Albrecht S, Golden SH. Depression and type 2 diabetes over the lifespan: a meta-analysis. Diabetes Care. 2008;31:2383-90.

16. Vancampfort D, Stubbs B. Physical activity and metabolic disease among people with affective disorders: prevention, management and implementation. J Affect Disord. 2016;224:87-94.

17. Mead G, Morley W, Campbell P, Greig C, Murdo MM, Lawlor D. Exercise for depression. Cochrane Database Syst Rev. 2009;(3):CD004366. https://doi. org/10.1002/14651858.CD004366.pub4.

18. Knubben K, Reischies FM, Adli M, Schlattmann P, Bauer M, Dimeo F. A randomised, controlled study on the effects of a short-term endurance training programme in patients with major depression. Br J Sports Med. 2007:41:29-33.

19. Martinsen E, Medhus A, Sandvik L. Effects of aerobic exercise on depression: a controlled study. BMJ. 1985;291(6488):109.

20. Schuch FB, Vasconcelos-Moreno MP, Borowsky C, Zimmermann AB, Rocha NS, Fleck MP. Exercise and severe major depression: effect on symptom severity and quality of life at discharge in an inpatient cohort. J Psychiatr Res. 2015;61:25-32.

21. Cooney G, Dwan K, Greig CA, Lawlor DA, Rimer J, Waugh FR, McMurdo M, Mead GE. Exercise for depression. Cochrane Database Syst Rev. 2013;(9):CD004366. https://doi.org/10.1002/14651858.CD004366.pub6.

22. Krogh J, Nordentoft M, Sterne JA, Lawlor DA. The effect of exercise in clinically depressed adults: systematic review and meta-analysis of randomized controlled trials. J Clin Psychiatry. 2011;72:529-38.

23. Excellence NIfHaC. Depression: the treatment and management of depression in adults. London: National Institute for Health and Clinical Excellence; 2009.
24. Ledochowski L, Stark R, Ruedl G, Kopp M. Physical activity as therapeutic intervention for depression. Nervenarzt. 2017;88:765-78.

25. Spedding S. Exercise for depression: Cochrane systematic reviews are rigorous, but how subjective are the assessment of bias and the practice implications? Adv Integr Med. 2015;2:63-5.

26. Kruisdijk FR, Hendriksen IJM, Tak ECPM, Beekman ATF, Hopman-Rock M. Effect of running therapy on depression (EFFORT-D). Design of a randomised controlled trial in adult patients. BMC Public Health. 2012;12:50.

27. Busch AM, Ciccolo JT, Puspitasari AJ, Nosrat S, Whitworth JW, StultsKolehmainen M. Preferences for exercise as a treatment for depression. Mental Health Phys Act. 2016;10:68-72.

28. Searle A, Calnan M, Lewis G, Campbell J, Taylor A, Turner K. Patients' views of physical activity as treatment for depression: a qualitative study. $\mathrm{Br} J$ Gen Pract. 2011;61:149-56.

29. Pickett K, Kendrick T, Yardley L."A forward movement into life": a qualitative study of how, why and when physical activity may benefit depression. Mental Health Phys Act. 2017;12:100-9.

30. Linnan L, Steckler A, editors. Process evaluation for public health, interventions and research. San Francisco: Jossey-Bass; 2002.

31. Firth J, Rosenbaum S, Stubbs B, Gorczynski P, Yung AR, Vancampfort D. Motivating factors and barriers towards exercise in severe mental illness: a systematic review and meta-analysis. Psychol Med. 2016;46:2869-81.

32. Krogh J, Saltin B, Gluud C, Nordentoft M. The DEMO trial: a randomized, parallel-group, observer-blinded clinical trial of strength versus aerobic versus relaxation training for patients with mild to moderate depression. J Clin Psychiatry. 2009;70:790-800.

33. Krogh J, Lorentzen AK, Subhi Y, Nordentoft M. Predictors of adherence to exercise interventions in patients with clinical depression - a pooled analysis from two clinical trials. Mental Health Phys Act. 2014;7(1):50-4.

34. Posternak MA, Miller I. Untreated short-term course of major depression: a meta-analysis of outcomes from studies using wait-list control groups. J Affect Disord. 2001;66:139-46.

35. Vancampfort D, Madou T, Moens H, De Backer T, Vanhalst P, Helon C, Naert P, Rosenbaum S, Stubbs B, Probst M. Could autonomous motivation hold the key to successfully implementing lifestyle changes in affective disorders? A multicentre cross sectional study. Psychiatry Res. 2015;228:100-6.

36. Firth J, Carney R, Pownall M, French P, Elliott R, Cotter J, Yung AR. Challenges in implementing an exercise intervention within residential psychiatric care: a mixed methods study. Mental Health Phys Act. 2017:12:141-6.

37. Fleischhacker WW, Cetkovich-Bakmas M, De Hert M, Hennekens CH, Lambert M, Leucht S, Maj M, McIntyre RS, Naber D, Newcomer JW, et al. Comorbid somatic illnesses in patients with severe mental disorders: clinical, policy, and research challenges. J Clin Psychiatry. 2008;69:514-9.

38. Vancampfort D, Rosenbaum S, Schuch F, Ward PB, Richards J, Mugisha J, Probst M, Stubbs B. Cardiorespiratory fitness in severe mental illness: a systematic review and meta-analysis. Sports Med. 2017;47(2):343-52.

39. Correll CU, Solmi M, Veronese N, Bortolato B, Rosson S, Santonastaso P, Thapa-Chhetri N, Fornaro M, Gallicchio D, Collantoni E, et al. Prevalence, incidence and mortality from cardiovascular disease in patients with pooled and specific severe mental illness: a large-scale meta-analysis of 3,211,768 patients and 113,383,368 controls. World Psychiatry. 2017:16:163-80.

40. Kruisdijk F, Deenik J, Tenback D, Tak E, Beekman AJ, van Harten P, Hopman-Rock M, Hendriksen I. Accelerometer-measured sedentary behaviour and physical activity of inpatients with severe mental illness. Psychiatry Res. 2017;254:67-74.

41. Schuch FB, Vancampfort D, Rosenbaum S, Richards J, Ward PB, Stubbs B. Exercise improves physical and psychological quality of life in people with depression: a meta-analysis including the evaluation of control group response. Psychiatry Res. 2016;241:47-54.

42. Krogh J, Videbech P, Thomsen C, Gluud C, Nordentoft M. DEMO-II trial. Aerobic exercise versus stretching exercise in patients with major depression—a randomised clinical trial. PLOS ONE. 2012:7:e48316.

43. Pajonk FG, WobrockT, Gruber O, Scherk H, Berner D, Kaizl I, Kierer A, Muller S, Oest M, Meyer T, et al. Hippocampal plasticity in response to exercise in schizophrenia. Arch Gen Psychiatry. 2010;67:133-43.

44. Lovell R, Wheeler BW, Higgins SL, Irvine KN, Depledge MH. A systematic review of the health and well-being benefits of biodiverse environments. J Toxicol Environ Health B Crit Rev. 2014;17:1-20. 
45. Fruhauf A, Niedermeier M, Elliott LR, Ledochowski L, Marksteiner J, Kopp $M$. Acute effects of outdoor physical activity on affect and psychological well-being in depressed patients - a preliminary study. Mental Health Phys Act. 2016;10:4-9.

46. Naslund JA, Aschbrenner KA, Bartels SJ. Wearable devices and smartphones for activity tracking among people with serious mental illness. Mental Health Phys Act. 2016;10:10-7.
47. Babyak M, Blumenthal JA, Herman S, Khatri P, Doraiswamy M, Moore K, Craighead WE, Baldewicz TT, Krishnan KR. Exercise treatment for major depression: maintenance of therapeutic benefit at 10 months. Psychosom Med. 2000;62:633-8.

\section{Submit your next manuscript to BioMed Central} and we will help you at every step:

- We accept pre-submission inquiries

- Our selector tool helps you to find the most relevant journal

- We provide round the clock customer support

- Convenient online submission

- Thorough peer review

- Inclusion in PubMed and all major indexing services

- Maximum visibility for your research

Submit your manuscript at

www.biomedcentral com/submit 